\title{
From Resection to Disconnection for Seizure Control in Pediatric Epilepsy Children
}

\author{
Jun Kyu Hwang, M.D., ${ }^{*}$ Dong-Seok Kim, M.D., Ph.D. \\ Department of Pediatric Neurosurgery, Severance Children's Hospital, Yonsei University College of Medicine, Seoul, Korea
}

Epilepsy surgery revealed dramatically improved seizure outcomes over medical therapy in drug-resistant epilepsy patients. Children with epilepsy, however, have multiple epileptic focuses which require multilobar resection for better seizure outcome. Multilobar resection has not only the several severe surgical complications, such as hydrocephalus and shunt-related craniosynostosis, due to intracranial volume reduction. Isolation method (disconnection surgery) was progressively studied over epileptic focus removal (resective surgery) for seizure control. This concept was first introduced for functional hemispherotomy, and its primary principle is to preserve the vital vascularized brain that is functionally disconnected from the contralateral healthy brain. Currently in most epilepsy centers, the predominant disconnection surgical methods, including functional hemispherotomy, are continually being refined and are showing excellent results. They allow the functional isolation of the hemisphere or multi-lobe, affected by severe epilepsy. This review describes recent findings concerning the indication, surgical technique, seizure outcome and complications in several disconnection surgeries including the functional hemispherotomy for refractory pediatric epilepsy.

Key Words : Functional hemispherotomy · Cerebral decortication · Epilepsy.

\section{INTRODUCTION}

Two-thirds of epilepsy cases may be controlled with monotherapy or poly-therapy of antiepileptic drugs, but one-third still remains intractable. Drug-resistant epilepsies may be surgically cured. The introduction of modern neuroimaging since the late 1980s, such as magnetic resonance imaging (MRI), fluorodeoxyglucose-positron emission tomography (FDG-PET), ictal and interictal single photon emission computed tomography (SPECT), and magnetoencephalography (MEG), makes the detection of epileptic foci and patients cur- able with resective surgery easy. After the first randomized controlled trial by Wiebe et al. ${ }^{43)}$ in 2001, epilepsy surgery revealed dramatically improved seizure outcomes over medical therapy in refractory temporal lobe epilepsy patients. Consensus guidelines recommended early surgical referral of drugresistant epilepsy patients ${ }^{10,15,16)}$. The problem is that more than $60 \%$ of pediatric epilepsies have multiple epileptic foci and they require multilobar resection for better seizure outcome. Despite low permanent morbidity ${ }^{35}$, multilobar resection has several severe surgical complications due to intracranial volume reduction, such as hydrocephalus and shunt-related cra-

\footnotetext{
- Received : January 24, 2019 •Revised : March 18, $2019 \bullet$ Accepted : March 21, 2019

- Address for reprints : Dong-Seok Kim, M.D., Ph.D.

Department of Pediatric Neurosurgery, Severance Children's Hospital, Yonsei University College of Medicine, 50-1 Yonsei-ro, Seodaemun-gu, Seoul 03722, Korea Tel : +82-2-2228-2150, Fax : +82-2-393-9979, E-mail : dskim33@yuhs.ac

*Current affiliation : Department of Neurosurgery, Asan Medical Center, Ulsan University College of Medicine, Seoul, Korea
} 
niosynostosis, in addition to $19 \%$ visual field defect $19 \%$ and $1 \%$ new motor deficit.

Researchers, therefore, began to investigate isolation (disconnection surgery) method instead of removing the epileptic foci (resective surgery) for seizure control. This concept was first introduced for functional hemispherotomy. The purpose of hemispherotomy is to functionally isolate the epileptogenic zone which is widely diffused to an entire hemisphere. Before the era of functional hemispherotomy, Dandy first carried out anatomical hemispherectomy for the treatment of gliomas in 1928 and reported that this surgery could be applied in epilepsy treatment in $1938^{28)}$. In 1950, Krynauw ${ }^{24)}$ reported that the anatomical hemispherectomy, involving ligation of the anterior and middle cerebral arteries and subsequent en bloc removal of the cerebral hemisphere, was effective in infantile hemiplegia cases. Subsequently, technical variations of less resective and more disconnecting hemispherotomy, such as hemidecortication has been described by Ignelzi and Bucy ${ }^{21)}$, and functional hemispherotomy by Rasmussen ${ }^{32}$. The aforementioned hemispherectomy methods, however, have not resolved the fundamental problem of intracranial volume loss, such as hydrocephalus, chronic subdural fluid collection, or superficial cortical hemosiderosis ${ }^{30)}$. In order to avoid severe complications of the hemispherectomy, functional hemispherotomy techniques weIre introduced in the 1990's, by Delalande et al. ${ }^{14)}$, Villemure and Mascott ${ }^{41)}$, Villemure and Daniel ${ }^{42)}$, and Schramm et al. ${ }^{36,37)}$, each with their own solution to achieve complete functional disconnection of the hemisphere. The principle of functional hemispherotomy is to preserve the vital vascularized brain that is functionally disconnected from contralateral healthy brain. Currently in most epilepsy centers, the predominant disconnection surgical methods, including functional hemispherotomy, are continually being refined and are showing excellent results. They allow functional isolation of the hemisphere or multi-lobe, affected by severe epilepsy.

\section{FUNCTIONAL HEMISPHEROTOMY}

\section{Indication for functional hemispherotomy}

Children with hemiplegic epilepsy, characterized by unilateral hemispheric pathology issuing in refractory epilepsy are potential candidates for hemispherotomy ${ }^{22,27)}$. Infantile hemiplegic epilepsy is not a single disease entity. A number of disorders such as congenital neuronal migrational defects (cortical dysplasia, hemimegalencephaly, hemiconvulsion-hemiplegia-epilepsy syndrome) and the destructive lesions on the unilateral hemisphere (congenital porencephaly, perinatal cerebrovascular accidents, Sturge-Weber syndrome, Rasmussen encephalitis) lead to intractable partial seizures and hemiparesis. Although wider resection or extensive disconnection is thought to result in optimum seizure control, these procedures increase the risk of neurological deficits as well as motor and mental problems. Thus, the surgical procedure for epilepsy must be selected by taking the pathology, semiology, developmental status, and age of the patient into consideration.

\section{Preoperative evaluation}

Presurgical evaluation includes 1) family and personal history; 2) complete seizure history : onset of epilepsy, semiology and frequency of seizures that are classified according to the ILAE classification ${ }^{44)}$; 3) neurologic examination, especially focused on motor and/or sensory-motor lateralized neurologic deficits (hemiparesis, unilateral hypotonic syndrome, and hemianopsia); 4) neuropsychologic examination, 5) 24-hour video-electroencephalography (EEG); and 6) neuroimage studies such as brain MRI, PET, and ictal and interictal SPECT.

\section{Surgical technique of functional hemispherotomy}

In order to completely isolate the pathologic hemisphere from the normal healthy hemisphere, anatomical hemispherectomy is the most certain method, when disregarding the surgical complications. For achieving the same complete disconnection with minimal complications as the anatomical hemispherectomy, technically four common goals are necessary : 1) disconnection of the cortico-thalamic tract (internal disconnection of the internal capsule and corona radiata); 2) resection of the medial temporal structures; 3 ) total corpus callosotomy; and 4) and disconnection of the oribito-frontohypothalamic tract (disruption of the frontal horizontal fibers $)^{29)}$. Two main surgical routes for functional hemispherotomy are lateral approach via the surgical route around the Sylvian fissure (Fig. 1A; peri-insular functional hemispherotomy) and vertical approach that reaches the lateral ventricle and then the corpus callosum from the brain vertex (transcortical or transcallosal transventricular functional hemispherotomy) 

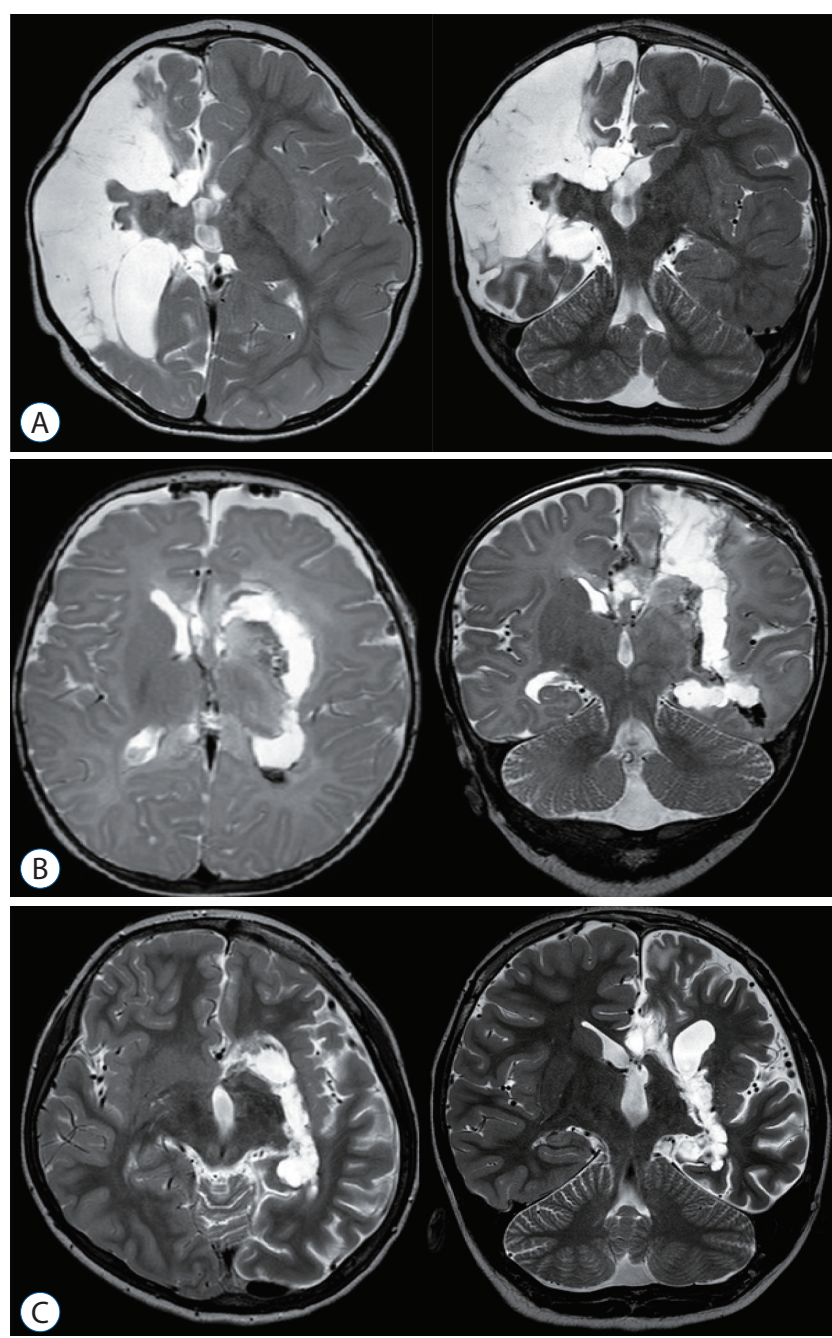

Fig. 1. Postoperative MRI of several functional hemispherotomies. A : Postoperative MRI of peri-insular functional hemispherotomy. B : Postoperative MRI of transcortical transventricular functional hemispheroctomy. C : Postoperative MRI of transcallosal transventricular functional hemispherotomy. $\mathrm{MRI}$ : magnetic resonance imaging.

(Fig. 1B and C).

The "peri-insular hemispherotomy"41,42) is divided into three surgical stages; supra-insular window, infra-insular window, and insula resection. Via the supra-insular window, the corpus callosum is reached to dissect the white matter of the corona radiata from the frontal and parietal cortex (cortico-thalamic tract) via the lateral ventricle preserving arteries and veins. After the callosotomy is completed along the pericallosal artery, posterior dissection extends from the hippocampus tail at the level of choroidal fissure to the fimbria-fornix. Anterior dissection extends from the fronto-basal portion just anterior to the basal ganglia via the suprainsular window.
The infra-insular window on superior temporal gyrus allows mesial temporal resection including the uncus, amygdala and hippocampus. Insular resection is completed by subpial aspiration by undermined incision at the level of the claustrum ${ }^{42)}$.

The general principle of "vertical parasagittal hemispherotomy" is to achieve the same line of disconnection as performed with the peri-insular hemispherotomy through a posterior frontal cortical window (Fig. 1B; transcortical transventricular hemispherotomy) or corpus callosum itself (Fig. 1C; transcallosal hemispherotomy $)^{14}$. The lateral ventricle with a small craniotomy can be reached identical to that of classic callosotomy. The dissection of the white matter of the corona radiata from the frontal and parietal cortex is done through the internal capsule lateral to the thalamus and continues from the trigone to the temporal horn of the lateral ventricle along the choroid plexus. Subsequently mesial temporal resection is performed. The surgery is completed with the disconnection of the fronto-basal portion just anterior to the basal ganglia. This surgical technique provides a favorable anatomical orientation and allows complete disconnection of the hemisphere with leaving the majority of the hemisphere intact along with its afferent and efferent vascular supply.

\section{Results : seizure outcome, cognition, and complica- tions}

Seizure outcome of hemispherectomy or hemispherotomy is favorable in patients with acquired lesions and patients with congenital malformations of cortical development with seizure free rate of $60-90 \%$ and a worthwhile improvement in $10-15 \%$ of patients ${ }^{1,36,41}$. Seizure outcomes, however, greatly differ according to the etiology and surgical techniques ${ }^{3,31}$. Rasmussen encephalitis, porencephaly secondary to perinatal stroke, Sturge-Weber syndrome have better prognosis than cortical malformations such as hemimegalencephaly and cortical dysplasia that may be associated with some degree of contralateral involvement ${ }^{14}$. Some authors pointed out that in terms of seizure outcome, anatomical hemispherectomy is most effective because of the early recurrence of seizures due to incomplete disconnection in functional hemispherotomy. Most neurosurgeons, however, agree that the complication rate is higher with anatomic hemispherectomy than with the more recent functional hemispherectomy or peri-insular hemispherotomy. Seizure outcome has been reported to be poorer in patients with cortical malformations compared to patients with acquired lesions. Unintended incomplete dis- 
connection is well-recognized surgical outcome of lateral or vertical functional hemispherotomy in patients with severe cortical malformations ${ }^{9,18}$. In patients with extensive cortical malformation, the technical constraints and difficulties involved in identifying anatomic hallmarks during the surgery may be the reason of incomplete disconnection. Incomplete basal ganglia disconnection may also play a significant role in seizure recurrence after hemispherotomy for patients with malformations of cortical development ${ }^{2,13,17)}$. Another reason for poorer seizure outcome in group with cortical malformations is their younger age at surgery. The underdeveloped brain of young children is too fragile to perform functional hemispherotomy which requires brain retraction during surgery. Poor surgical vision may lead to incomplete disconnection. Recently developed hemispherotomy concept, however, makes the seizure outcome quite similar with the three procedures; anatomical hemispherectomy, lateral or vertical functional hemispherotomy $y^{1,3,36,41)}$. Even if seizure free outcome does not depend on the surgical procedure, hemispherotomy techniques are highly recommended when insular and subcortical abnormalities are present ${ }^{25)}$.

Seizure outcome may sometimes predicted with preoperative EEG or MRI. Independent epileptic discharges from bilateral hemispheres indicate a less satisfactory outcome : in contrast abnormalities of background activity over the good hemisphere or bilaterally synchronous discharges may be associated with a good outcome ${ }^{39}$. In pre-operative MRI, contralateral abnormalities or abnormal hemispheres with extensive insular and subcortical heterotopic gray matter is also recognized as a poor predictive factor for seizure outcome $e^{6,7)}$. Moreover, contralateral MRI abnormalities may be observed in children with malformation of cortical development, affecting $25-72 \%{ }^{4,19}$. Nevertheless, contralateral abnormalities may not contraindicate hemispherotomy in order to decrease seizure frequency ${ }^{19,34)}$.

Post-surgery cognitive functions improve in most cases. Continuous epileptic discharges spreading from the malformed hemisphere to the "healthy" hemisphere suppress normal development of brain, which causes mental retardation ${ }^{8,40}$. Therefore, despite the remaining seizures, their frequency reduction allowed improvement in behavior, schoolwork and employment capabilities. The amount of improvement depends on the etiology, postoperative seizure freedom, duration of epilepsy before surgery and contralateral hemispheric dysfunction. A longer duration of epilepsy before surgery is associated with bad prognosis on global outcome, especially on verbal communications abilities ${ }^{3,8,11,14)}$. Post-surgery seizure freedom positively correlates with spoken language outcome in children with developmental etiology compared with acquired pathology $y^{11)}$. A lack of post-operative cognitive improvement may be related to abnormal metabolism as well as MRI abnormalities of the "healthy" hemisphere, ${ }^{8,19,33)}$.

Most patients who underwent hemispherotomy, suffered from transient aggravation of hemiparesis. Severe aggravation of hemiparesis or hemiplegia, however, has never occured in the long-term outcome ${ }^{3,14}$. Residual motor control is more severely impaired for hand functions than for walking ${ }^{11,14)}$.

Coagulopathy, aseptic meningitis, infections, cerebral infarction, hydrocephalus and superficial cerebral hemosiderosis are the early and delayed complications after surgery. The most common complication is hydrocephalus, which account for $10-50 \%$ of the cases ${ }^{9,14,20,42)}$, requires ventriculo-peritoneal or subduro-peritoneal shunting. Expected hemianopia is recorded in all operated patients. About $10 \%$ of cases require the second look surgery for seizure freedom because of persistent seizures and MRI evidence of incomplete disconnection ${ }^{3,5)}$. The perioperative mortality is higher than $1 \% \%^{9,14,20,42)}$. Early surgery allows better neurocognitive and psychosocial development providing several advantages in developmental plasticity, such as the transfer of motor functions in one hemisphere or language capability in the right hemisphere. Surgical consideration in younger children requires careful analysis of several age related issues in comparison to adults. Small blood volume and severe cortical malformations that occasionally need larger resection in infants may be closely related to the higher mortality rate ${ }^{20)}$. Rare mortality may happen in patient with Rasmussen encephalitis before severe atrophy, in SturgeWeber syndrome, in hemimegalencephaly or hypertrophic diffuse hemispheric dysplasia because of brain swelling and hemispheric infarct secondary to ischemia, from interfering with the arterial supply or venous drainage, which leads to intracranial hypertension and sudden death ${ }^{14)}$.

\section{POSTERIOR QUADRANTECTOMY}

\section{Indication for functional posterior quadrantec- tomy}

Although hemispherotomy is the treatment of choice when 

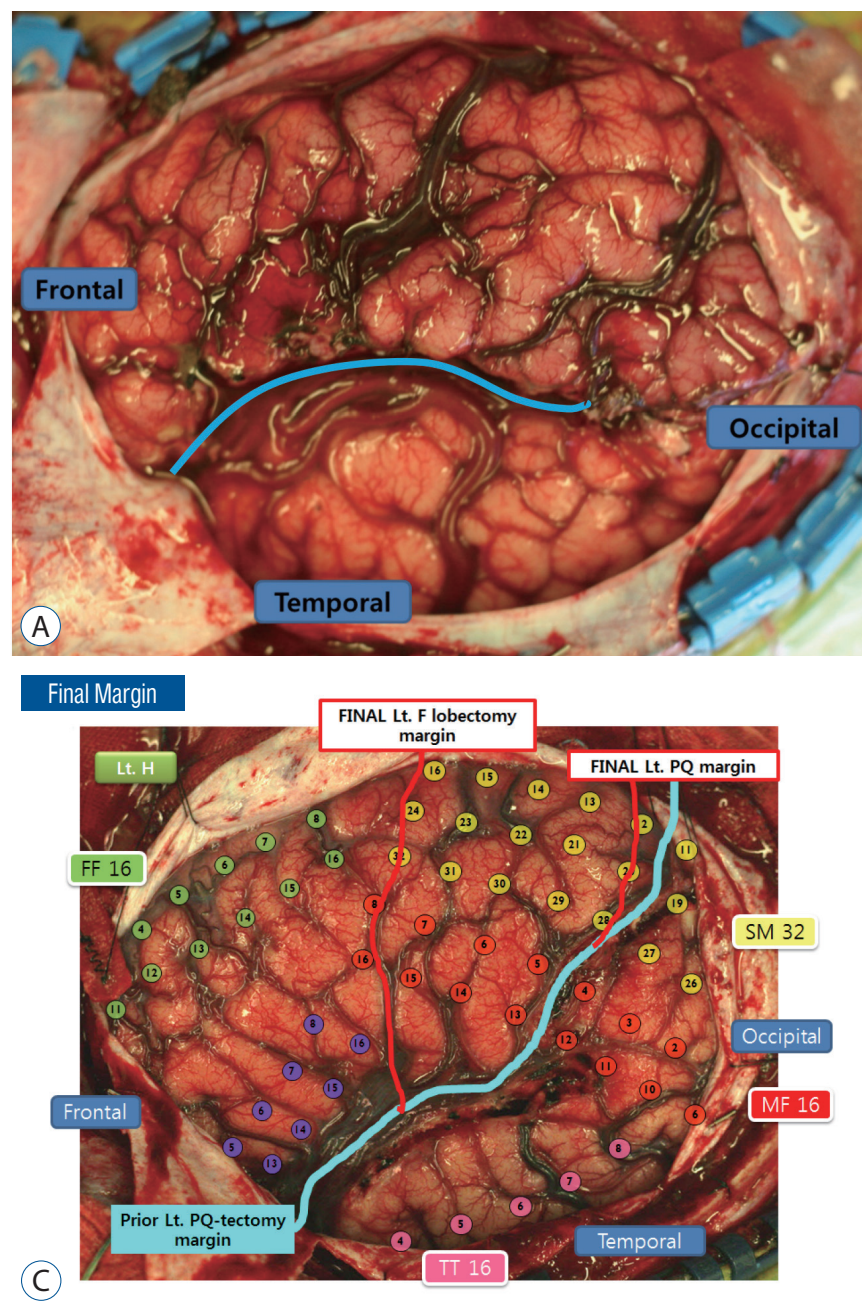

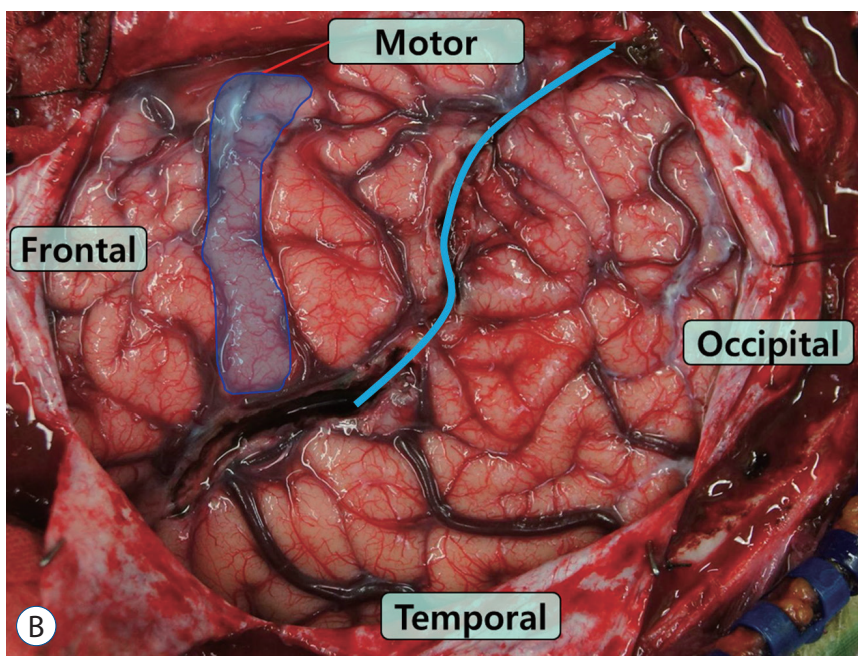

After Lt. frontal lobectomy

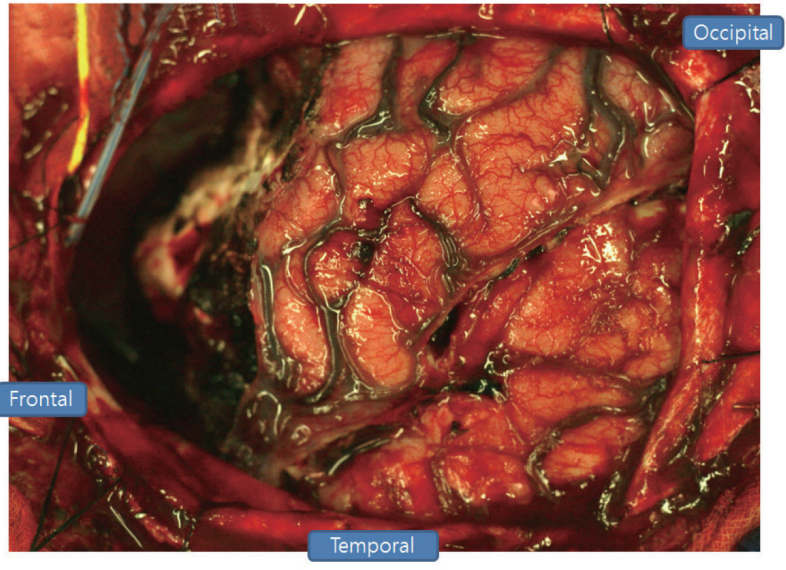

Fig. 2. Operative finding of several subhemispheric surgeries. A: Operative finding of anterior quadrantectomy. B: Operative finding of posterior quadrantectomy. C: Operative finding of posterior quadrantectomy, followed by frontal lobectomy. Final cortical incision margin (red line below) added on posterior quadrantectomy (light sea green line below). Lt. : left, $\mathrm{H}$ : hemisphere, $\mathrm{F}$ : frontal, $\mathrm{PQ}$ : posterior quadrantectomy.

the whole hemisphere is involved with accompanying hemiplegia, multilobar resection should be considered to spare the functional cortex with residual motor function (finger opposition and foot tapping) in sub-hemispheric involvement of epileptic zone. Multilobar epilepsy has traditionally been treated with resective surgery, which involves the removal of large parts of the hemisphere, either the frontal and parietal lobes (Fig. 2A; anterior quadrant resection), or the temporal, parietal, and occipital lobes (Fig. 2B; posterior quadrant resection), leaving behind a large operative cavity. Similar to the evolution of disconnective techniques in hemispherectomy, our surgical approach for quadrantic epilepsy has evolved progressively toward more disconnection and less resection. While maintaining similar seizure outcomes as resective sur- gery, these techniques are aimed at reducing perioperative morbidity and long-term complications. Quadrantectomy is indicated when the epileptogenic zone encompasses unilaterally large areas of the frontal and parietal lobes (anterior quadrant), or the temporal, parietal, and occipital lobes (posterior quadrant). At times posterior quadrantectomy can be considered followed by tailored frontal lobectomy (Fig. 2C) when epileptic zone involves the whole hemisphere without accompanying hemiplegia (Fig. 3). The pathological substrate responsible for the seizure disorder should be static, contrary to a progressive one like Rasmussen encephalitis, which may later necessitate hemispherectomy, even if only part of the hemisphere appears to be involved at its onset. The presence of residual voluntary motor function of the contralateral distal 


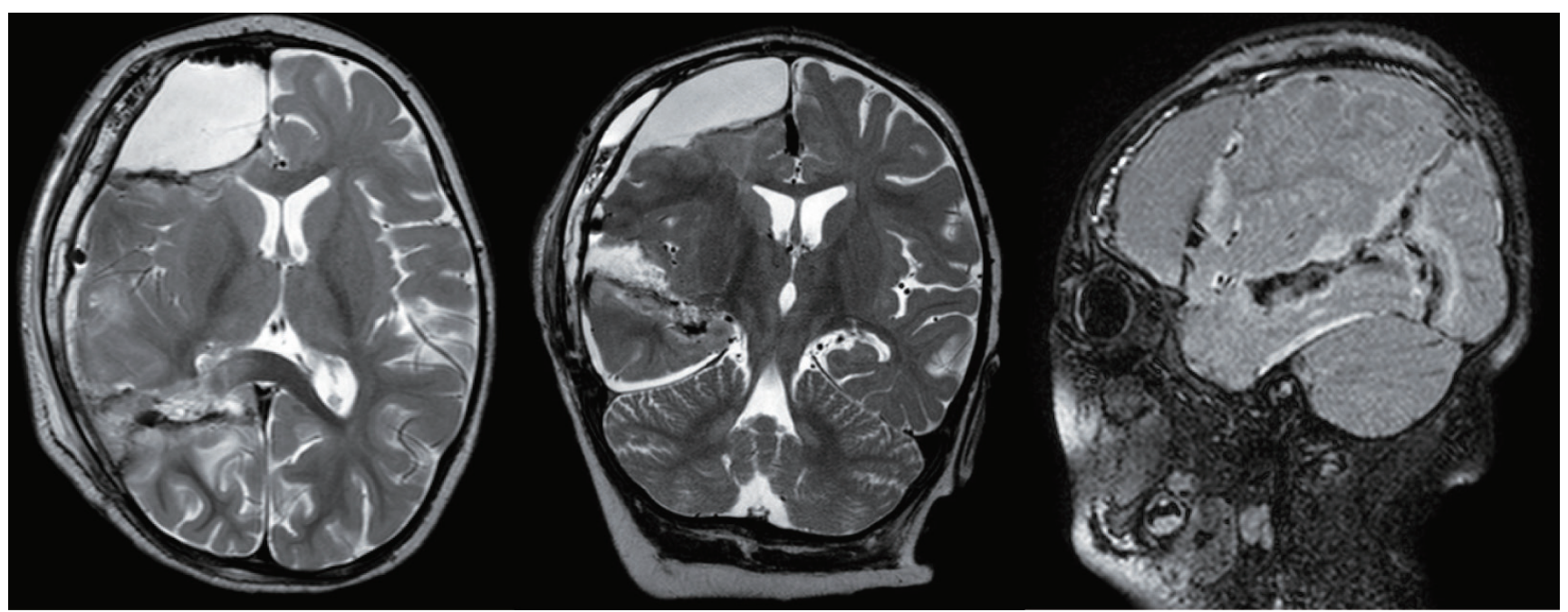

Fig. 3. Postoperative magnetic resonance imaging of posterior quadrantectomy, followed by frontal lobectomy.

musculature, i.e., finger opposition and foot tapping, contraindicates a hemispherectomy but forms the indication for posterior quadrantectomy. The presence of a visual field defect facilitates the decision to carry out this extensive surgery. However, the creation or aggravation of a homonymous field deficit is not an absolute contraindication to this surgery.

\section{Surgical technique of functional posterior quadran- tectomy}

The whole operation begins in a similar way to peri-insular hemispherotomy. A clear exposure of the opercular cortices and the central area (perirolandic) is essential for the surgery. The resection margin is tailored to encompass the whole epileptogenic lesion and to avoid the central region. The primary motor and sensory cortices are easily recognized from scrupulous study of the MRI, intraoperative surface anatomy, based on gyral pattern, arteries, and veins, and somatosensory evoked potentials or motor evoked potentials. This identification maximizes both the extent of resection and the safety of surgery.

The first step of functional posterior quadrantectomy is the removal of mesial temporal resection via infrainsular window. This step is identical to that of peri-insular hemispherotomy. The infra-insular window on superior temporal gyrus allows mesial temporal resection including the uncus, amygdala and the hippocampus. The proceeding step of the surgery is the disconnection of the neocortex of temporal, parietal and occipital lobes. The infrainsular window is posteriorly extended along the temporal horn to trigone of the lateral ventricle, taking care to preserve the vein of Labbe and the branches of the middle cerebral artery. The cortical incision in the superior temporal gyrus is turned around onto the suprasylvian cortex just posterior to the primary sensory cortex. The insular resection is completed by subpial aspiration by undermined incision at the level of the claustrum ${ }^{42}$. The third step is the intraparietal disconnection. The cortical incision is taken diagonally superiorly just posterior to the postcentral gyrus. The white matter along the whole length of the incision is deepened to reach the tissue along the falx and the disconnection is done up to the pia along the falx to reach the sagittal sinus superiorly and the level of the corpus callosum inferiorly. The last step is the posterior callosotomy (resection of splenum of corpus callosum). After completion of surgery, the temporal, parietal, and occipital lobe are anatomically left in situ but functionally completely isolated from ipsilateral frontal lobe and contralateral hemisphere.

\section{Results : seizure outcome and complications}

It is difficult to interpret surgical outcome of subhemispheric epilepsy because there are few studies that focus on this epilepsy surgery subgroup. Leiphart et al. ${ }^{26)}$ and Koszewski et al. ${ }^{23)}$ reported that the outcome of seizure after multilobal resections was inferior to that of single lobar resections. In contrast, Daniel et al. ${ }^{12)}$ reported that $92 \%$ of the cases showed no mortality or significant morbidity in Engel's class I. They pointed out that the incorporation of disconnective tech- 
niques in multilobar surgery has maintained the excellent results. Disconnective technique has markedly reduced the rate of hydrocephalus ${ }^{12,37)}$, a well-recognized complication ${ }^{12,32)}$ after hemispherectomy or multilobar subhemispheric resection. Endoscopic resection has been attempted even in the disconnection of small hypothalamic hamartoma. Some reports indicate endoscopic disconnection of hypothalamic hamartoma to be safer and more effective than other modalities ${ }^{38}$. Although such positive outcome has been marked with disconnective technique, it is a recent technique which lacks long term follow up. For all the other static etiologies, a disconnection surgery is a viable alternative option.

In the dominant posterior multilobar surgery, speech should be a major concern. For the confirmation of speech function, Wada test is needed. Fortunately, most of the language functions resided in the opposite hemisphere of the congenital lesions, resulting in the absence of postoperative language dysfunction.

\section{CONCLUSION}

Cerebral hemispheric disconnection surgery is a well-established treatment for intractable epilepsy. It is a secondary phenomenon that is diffuse, usually unilateral hemispheric, and intractable to medical therapy. Hemispherotomy or subhemispheric disconnection surgery may provide remarkable results in terms of seizure outcome and improvement of the quality of life. It is crucial for the epilepsy surgeon to appreciate the individuality of each patient who is a candidate for hemispheric or sunhemispheric disconnection and to apply the most suitable technique for each patient.

\section{CONFLICTS OF INTEREST}

No potential conflict of interest relevant to this article was reported.

\section{INFORMED CONSENT}

This type of study does not require informed consent.

\section{References}

1. Bahuleyan B, Manjila S, Robinson S, Cohen AR : Minimally invasive endoscopic transventricular hemispherotomy for medically intractable epilepsy: a new approach and cadaveric demonstration. J Neurosurg Pediatr 6 : 536-540, 2010

2. Basheer SN, Connolly MB, Lautzenhiser A, Sherman EM, Hendson G, Steinbok $P$ : Hemispheric surgery in children with refractory epilepsy: seizure outcome, complications, and adaptive function. Epilepsia 48 : 133-140, 2007

3. Battaglia D, Di Rocco C, luvone L, Acquafondata C, lannelli A, Lettori D, et al. : Neuro-cognitive development and epilepsy outcome in children with surgically treated hemimegalencephaly. Neuropediatrics 30 : 307-313, 1999

4. Boshuisen K, van Schooneveld MM, Leijten FS, de Kort GA, van Rijen PC, Gosselaar PH, et al. : Contralateral MRI abnormalities affect seizure and cognitive outcome after hemispherectomy. Neurology 75 : 16231630,2010

5. Bulteau C, Otsuki T, Delalande 0 : Epilepsy surgery for hemispheric syndromes in infants: hemimegalencepahly and hemispheric cortical dysplasia. Brain Dev 35 : 742-747, 2013

6. Carreño M, Wyllie E, Bingaman W, Kotagal $P$, Comair Y, Ruggieri P : Seizure outcome after functional hemispherectomy for malformations of cortical development. Neurology 57 : 331-333, 2001

7. Cats EA, Kho KH, Van Nieuwenhuizen O, Van Veelen CW, Gosselaar PH, Van Rijen PC : Seizure freedom after functional hemispherectomy and a possible role for the insular cortex: the Dutch experience. J Neurosurg 107(4 Suppl) : 275-280, 2007

8. Chiron C, Raynaud C, Jambaqué I, Dulac O, Zilbovicius M, Syrota A : A serial study of regional cerebral blood flow before and after hemispherectomy in a child. Epilepsy Res 8 : 232-240, 1991

9. Cook SW, Nguyen ST, Hu B, Yudovin S, Shields WD, Vinters HV, et al. : Cerebral hemispherectomy in pediatric patients with epilepsy: comparison of three techniques by pathological substrate in 115 patients. J Neurosurg 100(2 Suppl Pediatrics) : 125-141, 2004

10. Cross JH, Jayakar P, Nordli D, Delalande O, Duchowny M, Wieser HG, et al. : Proposed criteria for referral and evaluation of children for epilepsy surgery: recommendations of the Subcommission for Pediatric Epilepsy Surgery. Epilepsia 47 : 952-959, 2006

11. Curtiss S, de Bode S, Mathern GW : Spoken language outcomes after hemispherectomy: factoring in etiology. Brain Lang 79 : 379-396, 2001

12. Daniel RT, Meagher-Villemure K, Farmer JP, Andermann F, Villemure $J G$ : Posterior quadrantic epilepsy surgery: technical variants, surgical anatomy, and case series. Epilepsia 48 : 1429-1437, 2007

13. de Almeida AN, Marino R Jr, Marie SK, Aguiar PH, Teixeira MJ : Factors of morbidity in hemispherectomies: surgical technique $x$ pathology. Brain Dev 28 : 215-222, 2006

14. Delalande O, Bulteau C, Dellatolas G, Fohlen M, Jalin C, Buret V, et al. : Vertical parasagittal hemispherotomy: surgical procedures and clinical long-term outcomes in a population of 83 children. Neurosurgery 
60(2 Suppl 1) : ONS19-ONS32; discussion ONS32, 2007

15. Engel J Jr, Wiebe $S$ : Who is a surgical candidate? Handb Clin Neurol $108: 821-828,2012$

16. Engel J Jr, Wiebe S, French J, Sperling M, Williamson P, Spencer D, et al. : Practice parameter: temporal lobe and localized neocortical resections for epilepsy: report of the Quality Standards Subcommittee of the American Academy of Neurology, in association with the American Epilepsy Society and the American Association of Neurological Surgeons. Neurology 60 : 538-547, 2003

17. Gonzalez-Martinez JA, Gupta A, Kotagal P, Lachhwani D, Wyllie E, Luders $\mathrm{HO}$, et al. : Hemispherectomy for catastrophic epilepsy in infants. Epilepsia 46 : 1518-1525, 2005

18. Gowda S, Salazar F, Bingaman WE, Kotagal P, Lachhwani DL, Gupta A, et al. : Surgery for catastrophic epilepsy in infants 6 months of age and younger. J Neurosurg Pediatr 5 : 603-607, 2010

19. Hallbook T, Ruggieri P, Adina C, Lachhwani DK, Gupta A, Kotagal P, et al. : Contralateral MRI abnormalities in candidates for hemispherectomy for refractory epilepsy. Epilepsia 51 : 556-563, 2010

20. Holloway V, Gadian DG, Vargha-Khadem F, Porter DA, Boyd SG, Connelly $A$ : The reorganization of sensorimotor function in children after hemispherectomy. A functional MRI and somatosensory evoked potential study. Brain 123 Pt 12 : 2432-2444, 2000

21. Ignelzi RJ, Bucy PC : Cerebral hemidecortication in the treatment of infantile cerebral hemiatrophy. J Nerv Ment Dis 147 : 14-30, 1968

22. Kishima H, Oshino S, Tani N, Maruo Y, Morris S, Khoo HM, et al. : Which is the most appropriate disconnection surgery for refractory epilepsy in childhood? Neurol Med Chir (Tokyo) 53 : 814-820, 2013

23. Koszewski W, Czarkwiani L, Bidziński J : Multilobar resections in surgical treatment of medically intractable epilepsy. Neurol Neurochir Pol 32 Suppl $2: 81-94,1998$

24. Krynauw RA : Infantile hemiplegia treated by removing one cerebral hemisphere. J Neurol Neurosurg Psychiatry 13 : 243-267, 1950

25. Kwan A, Ng WH, Otsubo H, Ochi A, Snead OC 3rd, Tamber MS, et al. : Hemispherectomy for the control of intractable epilepsy in childhood: comparison of 2 surgical techniques in a single institution. Neurosurgery 67(2 Suppl Operative) : 429-436, 2010

26. Leiphart JW, Peacock WJ, Mathern GW : Lobar and multilobar resections for medically intractable pediatric epilepsy. Pediatr Neurosurg 34 : 311-318, 2001

27. Marras CE, Granata T, Franzini A, Freri E, Villani F, Casazza M, et al. : Hemispherotomy and functional hemispherectomy: indications and outcome. Epilepsy Res 89 : 104-112, 2010

28. McKenzie $\mathrm{K}$ : The present status of a patient who had the right cerebral hemisphere removed. JAMA 111 : 168-183, 1938

29. Morino M, Shimizu H, Ohata K, Tanaka K, Hara M : Anatomical analysis of different hemispherotomy procedures based on dissection of cadav- eric brains. J Neurosurg 97 : 423-431, 2002

30. Oppenheimer DR, Griffith HB : Persistent intracranial bleeding as a complication of hemispherectomy. J Neurol Neurosurg Psychiatry 29 : 229-240, 1966

31. Pinto AL, Lohani S, Bergin AM, Bourgeois BF, Black PM, Prabhu SP, et al. : Surgery for intractable epilepsy due to unilateral brain disease: a retrospective study comparing hemispherectomy techniques. Pediatr Neurol 51 : 336-343, 2014

32. Rasmussen $\mathrm{T}$ : Hemispherectomy for seizures revisited. Can J Neurol Sci $10: 71-78,1983$

33. Rintahaka PJ, Chugani HT, Messa C, Phelps ME : Hemimegalencephaly: evaluation with positron emission tomography. Pediatr Neurol 9 : 2128, 1993

34. Saito $Y$, Sugai $K$, Nakagawa $E$, Sakuma $H$, Komaki $H$, Sasaki $M$, et al. : Treatment of epilepsy in severely disabled children with bilateral brain malformations. J Neurol Sci 277 : 37-49, 2009

35. Sarkis RA, Jehi L, Najm IM, Kotagal P, Bingaman WE : Seizure outcomes following multilobar epilepsy surgery. Epilepsia 53 : 44-50, 2012

36. Schramm J, Behrens E, Entzian W : Hemispherical deafferentation: an alternative to functional hemispherectomy. Neurosurgery 36 : 509 515; discussion 515-506, 1995

37. Schramm J, Kuczaty S, Sassen R, Elger CE, von Lehe M : Pediatric functional hemispherectomy: outcome in 92 patients. Acta Neurochir (Wien) 154 : 2017-2028, 2012

38. Shim KW, Park EK, Kim DS, Choi JU : Neuroendoscopy: current and future perspectives. J Korean Neurosurg Soc 60 : 322-326, 2017

39. Smith SJ, Andermann F, Villemure JG, Rasmussen TB, Quesney LF : Functional hemispherectomy: EEG findings, spiking from isolated brain postoperatively, and prediction of outcome. Neurology 41 : 1790 1794, 1991

40. Soufflet C, Bulteau C, Delalande O, Pinton F, Jalin C, Plouin P, et al. : The nonmalformed hemisphere is secondarily impaired in young children with hemimegalencephaly: a pre- and postsurgery study with SPECT and EEG. Epilepsia 45 : 1375-1382, 2004

41. Villemure JG, Mascott CR : Peri-insular hemispherotomy: surgical principles and anatomy. Neurosurgery 37 : 975-981, 1995

42. Villemure JG, Daniel RT : Peri-insular hemispherotomy in paediatric epilepsy. Childs Nerv Syst 22 : 967-981, 2006

43. Wiebe S, Blume WT, Girvin JP, Eliasziw M; Effectiveness and Efficiency of Surgery for Temporal Lobe Epilepsy Study Group : A randomized, controlled trial of surgery for temporal-lobe epilepsy. N Engl J Med 345 : 311-318, 2001

44. Proposal for revised classification of epilepsies and epileptic syndromes. Commission on Classification and Terminology of the International League Against Epilepsy. Epilepsia 30 : 389-399, 1989 